\title{
Nonbacterial thrombotic endocarditis - Not forgotten
}

\author{
Sofia Metaxa MD ${ }^{1}$, Mario Petrou, $\mathrm{FRCS}^{2}$, Constantinos G Missouris MD, FRCP ${ }^{1,3}$
}

Metaxa S, Petrou M, Missouris CG. Nonbacterial thrombotic endocarditisNot forgotten. Curr Res Cardiol 2017;4(1):134-35.

We report the case of a 36 years old man who presented with an incidental echocardiographic finding of a $1.1 \times 0.8 \mathrm{~cm}$ mobile structure on the mitral valve associated with mild to moderate eccentric jet of mitral regurgitation. Surgical excision confirmed the diagnosis of nonbacterial thrombotic endocarditis (NBTE), formerly known as marantic endocarditis. The cardiolipin IgG and IgM antibodies and the lupus anticoagulant were strongly positive confirming the diagnosis of antiphospholipid syndrome. The patient was promptly anticoagulated.
NBTE is a rare condition associated with advanced mucin secreting malignant tumours and hypercoagulable states. It is associated with high incidence of embolic events the most frequent ones being cerebral; the diagnosis is usually reached at post-mortem. Our case clearly demonstrates that vegetations on cardiac valves may pose a diagnostic dilemma and surgical intervention may be needed to reach a diagnosis. We propose that in patients with high likelihood of NBTE a transthoracic echocardiogram should be considered to assess for the above diagnosis which carries a high risk of life threatening embolic events. Anticoagulation or surgical excision is likely to improve cardiovascular outcome.

Key Words: Hypertension; Depression; Anxiety; Echocardiography; Thrombophilia

\section{CASE PRESENTATION}

A 36 year old asymptomatic man was referred to our clinic for further assessment and management of hypertension. He denied fevers, weight loss, rigors, or sweating. He was on treatment for anxiety and depression, and chronic pain resulting from accidental division of the digital nerve of the right index finger and also the radial nerve. He was on treatment with Diclofenac, Gabapentin, Tramadol, Lansoprazole. Olanzapine, Venlafaxine and Perindopril $4 \mathrm{mg}$ od. On examination he was apyrexial, the pulse was $66 \mathrm{bpm}$ and the supine blood pressure was $159 / 109 \mathrm{~mm} \mathrm{Hg}$. The serum sodium was $141 \mathrm{mmol} / \mathrm{l}$, potassium $5.0 \mathrm{mmol} / \mathrm{L}$ and creatinine was 86 Imol/L. Furthermore, the $\mathrm{Hb}$ was $13.0 \mathrm{~g} / \mathrm{dl}$ and the CRP $9 \mathrm{mg} / \mathrm{L}$. The resting ECG confirmed sinus rhythm with rare unifocal ventricular ectopic beats. The transthoracic echocardiogram confirmed a $1.1 \times 0.8 \mathrm{~cm}$ mobile and echogenic structure on the atrial surface of the posterior mitral valve leaflet with mild to moderate eccentric jet of mitral regurgitation. There was mild left ventricular hypertrophy and the other valves were structurally normal. The above findings were also confirmed on transoesophageal echocardiography. In view of the diagnostic dilemma the patient underwent a surgical excision of the above structure, and the mitral valve was repaired with a bovine pericardial patch. He made an uneventful recovery. Histology, however, revealed a mass composed of fibrin and thrombus with occasional endothelial cells suggestive of non-bacterial thrombotic endocarditis (NBTE) or marantic endocarditis (Figure 1).

The cardiolipin IgG and IgM antibodies and the lupus anticoagulant were strongly positive confirming the diagnosis of antiphospholipid syndrome. The patient was promptly anticoagulated.

\section{DISCUSSION}

Non-infective thrombotic endocarditis (formerly known as marantic endocarditis) is a condition characterized by sterile vegetations on the endocardium and mainly affects the cardiac valves. It consists largely of fibrin and platelet aggregates and without an inflammatory response and is associated with numerous diseases including autoimmune disorders and in particular hypercoagulable states like SLE or in patients possessing the antiphospholipid antibody (aPL). Other conditions associated with NBTE include mucin producing adenocarcinomas, haematoligical malignancies, acquired immune deficiency syndrome, disseminated intravascular coagulation (DIC) and chronic conditions like tuberculosis, trauma from indwelling right sided cardiac catheters or central lines, snake bites, burns, overdose and late effect of thoracic

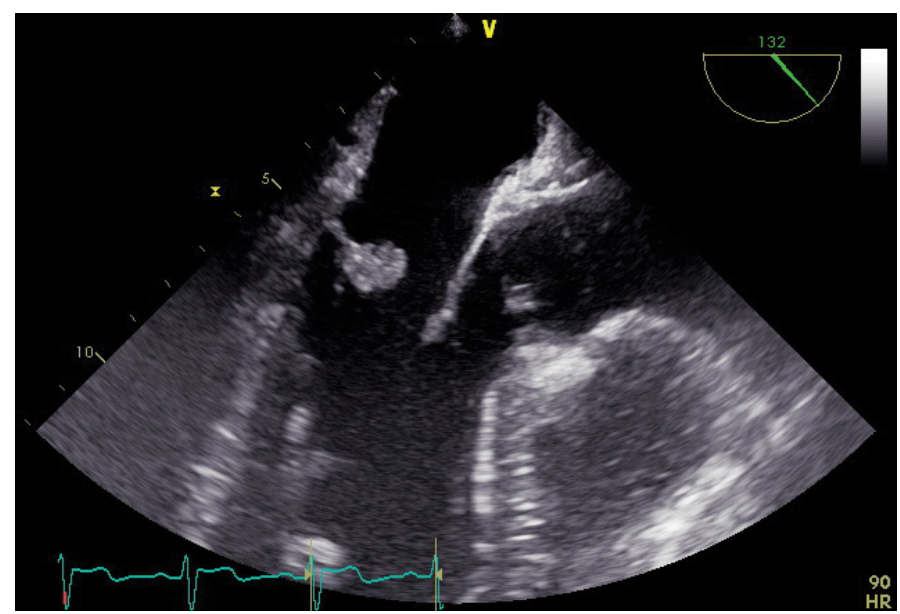

Figure 1) Transesophageal echocardiogram illustrating the intracardiac vegetation identified by a circle: it shows a large, nodular echodensity on the atrial aspect of the posterior mitral valve leaflet. AV Aortic valve; LA Left atrium; LV Left ventricle; RV Right ventricle

radiation treatment (1-3). There is no literature associating NBTE with administration of specific drugs (Figure 2).

Hypercoagulability with the associated venous or arterial thromboembolism was first described in 1865 by Trousseau as a presenting sign of malignancy (4). Adenocarcinomas of the lungs and the ovary constitute $50 \%$ of the cases and haematological malignancies account for approximately $25 \%$ (5). Zeigler in 1888 considered the lesions to be bland thrombi deposited on cardiac valves, and coined the word thromboendocarditis (6). This was subsequently described as marantic endocarditis due to its association with malignant and wasting diseases (7). At autopsy Libman and Sacks in 1924 described the above findings in a large number of patients with systemic lupus erethymatosus(up to 40\%). Gross and Friedberg in 1936 subsequently used the word nonbacterial thrombotic endocarditis (8). Angrist and Marquiss described the association between systemic emboli and this condition likely to be the result of either the superficial nature of these lesions or thelack of cellular organization (9). The diagnosis its mostly reached at post-mortum with an incidence of between $0.3 \%$ to $9.3 \%$ in the adult population $(10,11)$.

${ }^{1}$ Frimley Health NHS Foundation Trust and ${ }^{2} J o h n$ Radcliffe NHS Foundation Trust, ${ }^{3}$ University of Cyprus Medical School, Nicosia, Cyprus

Correspondence: Dr Constantinos G Missouris, University of Cyprus Medical School, Nicosia, Cyprus, Telephone +44 (0)2070794217, Fax +44 (0)207580 0122, e-mail dinos. missouris@fhft.nhs.uk

This open-access article is distributed under the terms of the Creative Commons Attribution Non-Commercial License (CC BY-NC) (http:// creativecommons.org/licenses/by-nc/4.0/), which permits reuse, distribution and reproduction of the article, provided that the original work is properly cited and the reuse is restricted to noncommercial purposes. For commercial reuse, contact reprints@pulsus.com 


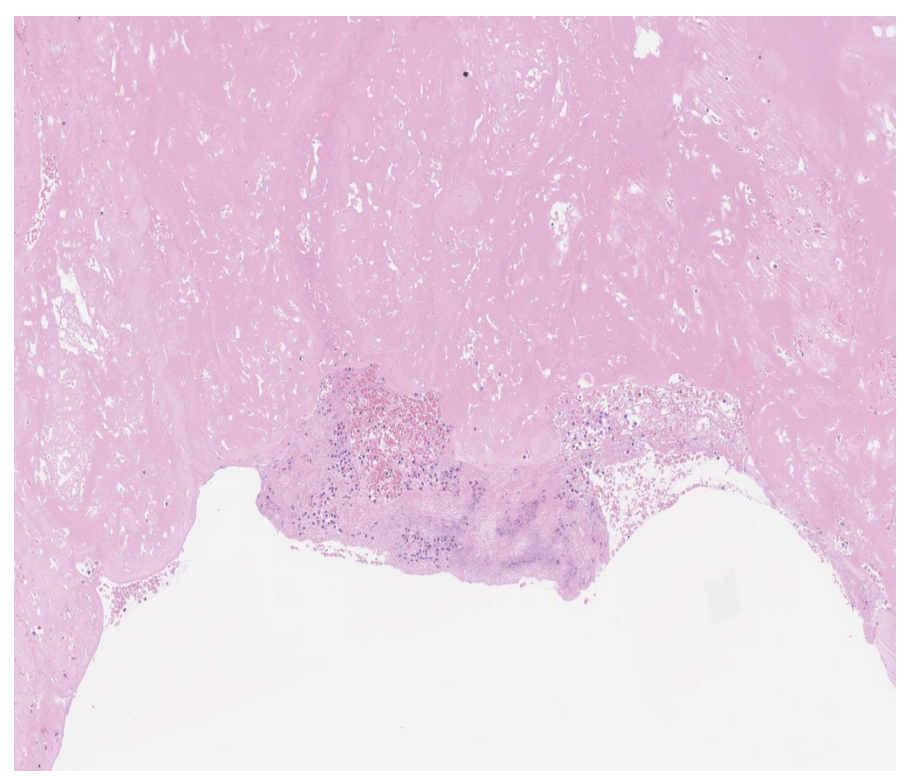

Figure 2) Photomicrograph of marantic vegetation of the mitral valve showing fibrin (amorphous pink deposits) predominantly with occasional inflammatory cells (blue cells) and erythrocytes (red cells), smooth lined thrombus throughout with no evidence of infection or neoplasm, stained with hematoxylin and eosin (original magnification $x 200)$

Furthermore, the incidence of visceral embolism (the majority being cerebral) is between $14-90 \%$ (12).

NBTE commonly affects those between the 4th and 8th decades of life and the diagnosis can be confused with culture negative endocarditis or the presence of fibroelastoma on the cardiac valves. With the advent of echocardiography more asymptomatic lesions are being diagnosed during life. The vegetations are mostly located on the left-sided heart valves (majority on the mitral valve) and cases of NBTE involving the right-sided heart valves are rare. The mural endocardium, chordae tendinae and papillary muscles may also be involved. Atrial myxomas or papillomas rarely arise from the cardiac valves. Fibroelastomas on the other hand usually arise from the surface of the valves and represent $<10 \%$ of primary cardiac tumours.

There are no ante mortem clinical findings or pathognomonic signs and symptoms that allow for NBTE and the diagnosis is made either at postmortum (13) or as an incidental finding on echocardiography. Negative blood cultures, the absence of clinical signs of infection and a normal thrombophilia screen are also important clues. Spontaneous venous thromboembolism is also more likely to occur in the presence of NBTE. However, the presence of a disease process known to be associated with NBTE, the presence of a heart murmur or the presence multiple systemic emboli (in several cerebral territories on diffusion-weighted MRI) may point towards the above diagnosis. On the other hand single or focal lesions and limited infarction are more characteristic of infective endocarditis. Both transthoracic ad transcoesophageal echocardiography have revolutionized the ante mortum diagnosis of NBTE. Characteristically valvular vegetations along the co-aptation lines, at the valve rings and at the commissures, and without overt destruction of valvular tissue are more suggestive of the above diagnosis. Some lesions on the cardiac valves, however, are indistinguishable from vegetations resulting from infective endocarditis.

The management of NBTE relies on the correction of the underlying disease process. Anticoagulation with vitamin $\mathrm{K}$ antagonists remains the mainstay treatment for NBTE in patients with SLE and positive antiphospholipid antibodies. In patients with malignancy and associated DIC, with the use of intravenous heparin is supported by the available evidence. In this group of patients the use of warfarin is not as effective (14-16). Furthermore, in patients with malignancy $(17,18)$ and asymptomatic valvular vegetations, but without DIC, the role of anticoagulation has not been proven. and the use of either warfarin or heparin should be balanced against the bleeding risks. Surgical excision of the vegetations may be considered in those patients with potentially curable cancers (19-21) in those with severe regurgitant valves, recurrent thromboembolic events (22) despite adequate anticoagulation and, as a clinical case demonstrates, in those patients where the diagnosis is uncertain. Surgical preservation of the affected valve should be the preferred surgical approach.

NBTE is a rare condition associated with advanced mucin secreting malignant tumours and hypercoagulable states. It is associated with high incidence of embolic events. The diagnosis is usually reached at postmortum. Our case clearly demonstrates that marantic vegetations on the cardiac valves may occur without the above diagnoses and pose a diagnostic dilemma. We propose that in patients with high likelihood of NBTE a transthoracic echocardiogram should be considered to assess for the above diagnosis. Surgical intervention may expedite the diagnosis and significantly reducing the risk of life threatening embolic events.

\section{REFERENCES}

1. Libman E, Sacks B. A hitherto undescribed form of valvular and mural endocarditis. Arch Intern Med 1924;33:701-37.

2. Katsouli A, Massad MG. Current issues in the diagnosis and management of blood culture-negative infective and non-infective endocarditis. Ann Thorac Surg 2013;95:1467-74.

3. Khorana AA, Malignancy, thrombosis and Trousseau the case foraneponym J Thromb Haemost 2003;1:2463-65.

4. Trousseau A. Phlegmasia alba dollens. Clin Ned Hotel Dieu De Paris 1965;3:94.

5. Bedikian A, Valdivieso M, Luna M. Nonbacterial thrombotic endocarditis in cáncer patients: comparison of characteristics of patients with and without concomitan disseminated intravascular coagulation. Med Pediatr Oncol 1978;4:149-57.

6. Zeigler R. Uber den bau die entstehungendocartischerefflorescenzen, WerhDtsch Kong. InternMed 1888;7:399.

7. Harbitz F. Studenuber endocarditis. Dtsch Med Wochenschr 1899;25:121.

8. Gross L, Friedberg CK. Nonbacterial thrombotic endocarditis. Classification and general describtion. Arch Intern Med 1936;58:620-40.

9. Angrist A, Marquiss J. The changing norphologic picture of endocarditis since the advent of chemotherapy and antibiotic agents. Am J Pathol 1954:30:39-63.

10. Steiner I. Nebakrerialnitrombotickaendokarditida-studie 171 pripadu (nonbacterialthrombotic endocarditis-a study of 171 case reports) Cesk Parol 1993;29:58-60.

11. Kuramoto K, Matsushita S, Yamanouchi H. Non-bacterial thrombotis endocarditis as a cause of cerebral and myocardial infarction. Jpn Circ J 1984;48:1000-06.

12. Lopez A, Ross R, Fishbein MC, et al. Nonbacterial thrombotic endocarditis: A review. Curr in Cardiol 1986;773-83.

13. Llenas-Garcia, Guerra-Vales JM, Montes-Moreno S, et al. Non-bacterial thrombotic endocardtis: clinico-patholocic study of necropsy series. RevEspCardiol 2007;60:493-500.

14. Sack GH, Levin J, Bell WR. Trousseau's syndrome and other manifestations of chronic disseminated coagulopathy in patients with neoplasms: clinical, pathophysiologic and therapeutic features. Medicine 1977;56:1-37.

15. Mosesson MW, Coleman RW, Sherry S. Chronic intravascular syndrome. N Engl J Med 1968;278:815-21.

16. Kazmier FJ, Bowie EJW, Hagedorn AB, et al. Treatment of intravascular coagulation and fibrinolysis (ICF) syndrome. Mayo ClinProc 1974;49:665-72.

17. Schlittler LA, Dallagasperina VW, Schavinski C, et al. Marantic endocarditis and adenocacinoma of unknown primary site. ArqBrasCardiol 2011;96:e73-5.

18. Mazokopakis EE, Syros PK, Starakis IK. Nonbacterial thrombotic endocarditis (marantic endocarditis) in cáncer patients. Cardiovasc Hemato Disord Drug Targets 2010;10:84-6.

19. Bick RL. Cancer associated thrombosis. N Engl J Med 2003;349:109-11.

20. Tasi SH, Juan CJ, Dai MS. Trousseau's síndrome related to adenocarcinoma of the colon and cholangiocarcinoma. Eur J Neurol 2004;11:493-6.

21. Suzuki S, Tanaka K, Nogawa S. Expression of interleukin-6 in cerebra neurons and ovarian cáncer tissue in Trousseau syndrome. ClinNeuropathol 2002;21:232-5.

22. Mukai M, Hamada M, Hiwada K, et al. Cerebral and myocardial infarction induced by nonbacterial thrombotic endocarditis in a patient with ovarian cáncer-report of a case. Jpn J Med 1988;27:321-4. 